\title{
COVID-19 und Plastische Chirurgie - Schönheitschirurgie oder wichtiger Bestandteil der medizinischen Versorgung?
}

\author{
Die Auswirkungen der COVID-19 Pandemie auf die Patientenversorgung an einer \\ plastisch - chirurgischen Abteilung eines Universitätsklinikums
}

\section{COVID-19 and plastic surgery: aesthetic surgery or essential medical care?}

Impact of the COVID-19 pandemic on patient care in the plastic surgery department at an university hospital

(c) $(1) \odot$

\begin{abstract}
Autoren
Daniel Popp ${ }^{\mathbb{D}}$, Christian Smolle, Sebastian P. Nischwitz, Isabelle Sawetz, Caroline Schaunig, Raimund Winter, Stephan Spendel, Lars-Peter Kamolz

Institut

Klinische Abteilung für Plastische, Ästhetische und Rekonstruktive Chirurgie, Universitätsklinik für Chirurgie, Medizinische Universität Graz

Schlüsselwörter

COVID-19, SARS-CoV-2, Plastische Chirurgie, Pandemie, Auswirkungen, Impact

Key words

Plastic surgery, pandemic, impact

eingereicht $\quad 07.07 .2020$

akzeptiert $\quad 21.10 .2020$

Bibliografie

Handchir Mikrochir Plast Chir 2021; 53: 185-193

Online-Publikation: 02.02.2021

DOI 10.1055/a-1308-2638

ISSN 0722-1819

(C) 2021. The Author(s).

This is an open access article published by Thieme under the terms of the Creative Commons Attribution-NonDerivative-NonCommercial-License, permitting copying and reproduction so long as the original work is given appropriate credit. Contents may not be used for commercial purposes, or adapted, remixed, transformed or built upon. (https://creativecommons.org/licenses/by-nc-nd/4.0/) Georg Thieme Verlag KG, Rüdigerstraße 14, 70469 Stuttgart, Germany

\author{
Korrespondenzadresse \\ Dr. med. univ. Daniel Popp \\ Klinische Abteilung für Plastische, Ästhetische \\ und Rekonstruktive Chirurgie \\ Universitätsklinik für Chirurgie \\ Medizinische Universität Graz \\ Auenbruggerplatz 29 \\ A-8036 Graz \\ Österreich \\ Tel.: +43 (316)-385-14685
}

E-Mail: daniel.popp@medunigraz.at

\section{ZUSAMMENFASSUNG}

Einleitung Im Frühjahr 2020 machte die COVID-19-Pandemie weitreichende Veränderungen und Maßnahmen in bisher nie dagewesenem Ausmaß notwendig. Diese Maßnahmen zielten einerseits darauf ab die Ausbreitung der Virusinfektion einzudämmen, und andererseits die landesweite Krankenversorgung weiterhin zu gewährleisten, insbesondere um im Falle einer größeren Anzahl an mit COVID-19 infizierten Patienten ausreichend Intensivkapazitäten zur Verfügung zu haben. In Bezug auf operative Fächer bedeutete dies das temporäre Aussetzen des Elektivprogramms auf unbestimmte Zeit. Ziel dieser Studie war es die Auswirkung dieser Maßnahmen auf das Behandlungsspektrum eines plastisch-chirurgischen Zentrums zu evaluieren. Methode Die Operationszahlen der klinischen Abteilung für Plastische, Ästhetische und Rekonstruktive Chirurgie der medizinischen Universität Graz während des sog. Lockdowns von 16.3.2020 bis 26.4.2020 (6Wochen) sowie 2 Wochen davor wurden retrospektiv erhoben. Die Daten wurden zum korrespondierende Vorjahreszeitraum verglichen. Untersucht wurden u. a. Operationsspektrum, Dringlichkeit, Indikation und Komplikationsraten.
\end{abstract}


Ergebnisse Durch den Wegfall des Elektivprogramms kam es während des Lockdowns im Jahresvergleich zu einem signifikanten Rückgang der OP-Zahlen um 57,5 \% (2019: 353, 2020150 Fälle). Während des Lockdowns wurden im Jahresvergleich signifikant mehr Notfall- und Akuteingriffe durchgeführt (2019: 41, 2020: 58 Fälle, p<0,001). Außerdem wurde eine signifikante Häufung von Selbstverletzungen bzw. Suizidversuchen beobachtet (2019: 0, 2020: 16 Fälle, p>0,001). Hinsichtlich Privat- und Arbeitsunfällen gab es keine signifikanten Unterschiede. Es zeigte sich auch kein signifikanter Unterschied bezüglich der Komplikationsrate (2019: 6,8, 2020: 10\%, p=0,219). Zusammenfassung Ein signifikanter Anteil plastisch-chirurgischer Operationen an einem Universitätsklinikum mit überregionalem Versorgungsauftrag besteht aus Notfalloperationen, akuten und dringenden medizinisch notwendigen Operationen. Während des Lockdowns wurden Operationen ohne signifikanten Anstieg der Komplikationsrate durchgeführt. Trotz sämtlicher Herausforderungen während der Pandemie konnte eine qualitativ konstante Patientenversorgung auf höchstem Niveau gewährleistet werden. Um die während des Lockdowns angehäuften weniger dringenden, jedoch ebenso wichtigen Fälle zeitgerecht und in entsprechend hoher Qualität abzuarbeiten, wird eine Erweiterung der Operationskapazitäten mit Steigerung der Bettenanzahl und der Ambulanzkapazität notwendig sein. Die vorliegenden Ergebnisse zeigen, wie wichtig die Plastische Chirurgie für die medizinische Versorgung insbesondere in Krisenzeiten ist.

\section{ABSTRACT}

Introduction In spring 2020, the COVID-19 pandemic required far-reaching changes and measures of unprecedented extent. These measures were implemented to reduce virus spread and to ensure the continuity of nation-wide medical care, in particular with a view to having sufficient intensive-care capacities in case of a large caseload of patients infected with
COVID-19. With regard to surgical specialties, this implied a temporary hold on elective cases for an indefinite period of time. The aim of this study was to assess the impact of these measures on the caseload of a level-three plastic surgery unit. Methods This study retrospectively assessed the caseload at the Division of Plastic, Aesthetic and Reconstructive Surgery at Medical University Graz during the so-called lockdown from 16 March 2020 to 27 April 2020 (6 weeks) as well as two weeks before. The data was compared with the corresponding time period of the year 2019. Surgical spectrum, procedural urgency, medical indication of surgical procedures as well as complication rates were compared.

Results The suspension of elective cases led to a significant reduction in caseload of $57.5 \%$ (2019: 353, 2020: 150 cases). There was a significant increase in emergency and acute case procedures performed during the lockdown compared with the previous year (2019: 41, 2020: 58 cases, $p<0.001$ ). Furthermore, the number of self-inflicted injuries and suicide attempts increased significantly (2019: 0, 2020: 16 cases, $p<0.001$ ). With regard to private and work-related injuries, there was no significant difference. Also, there was no difference in complication rates (2019: 6.8, 2020: 10\%, p=0.219).

Conclusion A significant amount of surgical procedures in plastic surgery at a supraregional academic health centre consists of emergency, acute and urgent medically necessary cases. During the lockdown, surgical procedures were performed without a significant increase in complication rates. Despite challenges during the pandemic, high-quality patient care was provided throughout. To process less urgent yet important cases accumulated during the lockdown in a reasonable amount of time and maintaining the same level of high-quality care, additional capacities regarding operating rooms, hospital beds and outpatient care are needed. These results point out the importance of plastic surgery for medical care, in particular during times of crisis.

\section{Einleitung}

Das erste Halbjahr 2020 war weltweit geprägt durch das Neuartige Coronavirus (SARS-CoV-2) und die dadurch verursachte Pandemie. Vielerorts machte die Pandemie rigorose Maßnahmen erforderlich, die allesamt zum Ziel hatten die Ausbreitung der Infektionskrankheit einzudämmen. Regierungen waren gezwungen ad hoc Notfallpläne zu erstellen und sahen sich mit der Herausforderung konfrontiert eine Balance zwischen kollektiven Maßnahmen wie der weitgehenden Einschränkung des öffentlichen Lebens, sowie der Drosselung des Gesundheitswesens auf der einen und Aufrechterhaltung der dringlichen Krankenversorgung auf der anderen Seite zu finden. Dabei wurde insbesondere versucht eine Überlastung der Versorgungskapazitäten, besonders im intensivmedizinischen Bereich zu verhindern.

Vom ersten nachgewiesenen COVID-19 Fall bis zur Umsetzung des im Österreichischen Nationalrat beschlossenen sogenannten 1. COVID-19-Gesetz („Bundesgesetz betreffend vorläufige Maßnahmen zur Verhinderung der Verbreitung von COVID-19“) dauerte es exakt drei Wochen. Am 16.3.2020 ist in Österreich anlässlich der raschen Ausbreitung des Corona-Virus und der rapiden Zunahme der Erkrankungsfälle bundesweit ein Maßnahmenpaket in Kraft getreten, das zum Ziel hatte die rasche Ausbreitung der Viruserkrankung einzudämmen und so eine Überlastung des Gesundheitssystems zu vermeiden. Die Maßnahmen beinhalteten zum einen zahlreiche Ausgangsbeschränkungen für Privatpersonen, zum anderen wurden viele Bereiche des öffentlichen- und Berufslebens eingeschränkt [1, 2].

In den österreichischen Krankenhäusern wurde zeitgleich auf Anordnung der Bundesregierung und der Landesregierungen der tägliche Betrieb auf ein Minimum gedrosselt, um im Bedarfsfall genügend Versorgungskapazität für hospitalisations- bzw. intensivpflichtige COVID-19 erkrankte Patienten zur Verfügung zu haben. Im Hinblick auf chirurgische Disziplinen bedeutete dies eine Stornierung elektiver Eingriffe mit sofortiger Wirkung bis auf Weiteres [3].

Eine Woche nach Inkrafttreten der österreichischen Maßnahmen erfolgte ein ähnliches Vorgehen in Deutschland. Im Unterschied zu 
Österreich wurde die Entscheidung über entsprechende Maßnahmen gemäß der Föderalstruktur jedoch der jeweiligen Landesregierung übertragen, weswegen hier kein einheitliches Vorgehen zu beschreiben ist. In weiterer Folge zeigte sich sowohl in Österreich als auch in Deutschland, welche insgesamt ähnliche Strategien verfolgten, im europäischen Vergleich relativ geringe Mortalitätsraten in Folge von COVID-19-Infektionen, sodass grundsätzlich von einem erfolgreichen Vorgehen, bzw. einem funktionierenden Notfallplan der entsprechenden Gesundheitssysteme auszugehen ist [4].

Entgegen der Wahrnehmung der Plastischen Chirurgie in der breiten Öffentlichkeit übernehmen plastisch-chirurgische Abteilungen an öffentlichen Spitälern als Letztversorger einen maßgeblichen Anteil in der Krankenversorgung, wobei der Schwerpunkt im plastisch-rekonstruktiven Behandlungsspektrum liegt [5-8]. Dazu zählen unter anderem die Versorgung komplexer Handverletzungen inklusive Replantationen, die Behandlung Schwerbrandverletzter, die chirurgische Therapie akuter Weichteil- und Knocheninfektionen sowie die interdisziplinäre Tumorchirurgie [9] - insbesondere im Falle der untersuchten universitären Abteilung in Graz mit überregionalem Versorgungsauftrag und einem dadurch entstehenden Einzugsgebiet mit über 2000000 Einwohnern. Dennoch wird angenommen, dass ein Wegfall elektiv chirurgischer Eingriffe insbesondere in der plastischen Chirurgie zu einem merklichen Rückgang der Fallzahlen geführt hat.

Ziel der Studie war es zu untersuchen, welche Auswirkungen die COVID-19-bedingten Maßnahmen auf Operationszahlen, Behandlungsspektrum und Komplikationen hatten und welche Folgen daraus abzuleiten sind. Daraus könnten richtungsweisende Informationen gewonnen werden, um für zukünftige Krisenszenarien besser vorbereitet zu sein. Zum Vergleich wurde der korrespondierende Zeitraum des Vorjahres herangezogen.

Außerdem soll damit die Wahrnehmung der Plastischen Chirurgie als „Schönheitschirurgie“ reduziert und ein stärkeres öffentliches Bewusstsein für die Plastische Chirurgie als das geschaffen werden, was sie tatsächlich ist: Ein essenzieller, integraler Bestandteil der medizinischen Versorgung, insbesondere in Krisenzeiten.

\section{Methoden}

\section{Daten}

Die Anzahl aller zwischen dem 2.3.2020 (Kalenderwoche 10, 2 Wochen vor dem Lockdown) und dem 26.4.2020 (6 Wochen nach dem Beginn des Lockdowns, Kalenderwoche 17) an der klinischen Abteilung für Plastische, Ästhetische und Rekonstruktive Chirurgie der Universitätsklinik für Chirurgie an der medizinischen Universität Graz durchgeführten Operationen wurde retrospektiv anhand der hausinternen OP-Dokumentation erhoben. Um die Auswirkungen des Lockdowns auf die Operationszahlen quantifizieren zu können, wurde zum Vergleich der korrespondierende Zeitraum des Vorjahres herangezogen.

\section{Erhobene Parameter}

Inkludiert wurden sämtliche Operationen, die im stationären, tagesklinischen oder ambulanten Setting in Allgemein-, Regionaloder Lokalanästhesie durchgeführt wurden.
Folgende patientenspezifische Parameter wurden erhoben: Alter, Geschlecht, Unfall-Anamnese (Privatunfall, Arbeitsunfall bzw. absichtliche Selbstverletzung/Suizidversuch vs. kein Unfall) und ASA-Status.

Die Art der durchgeführten Eingriffe wurde erhoben und entsprechend dem plastisch-chirurgischen 4-Säulen Modell der Verbrennungschirurgie, Handchirurgie, Ästhetischen oder Rekonstruktiven Chirurgie zugeordnet. Zusätzlich wurden die Eingriffe nach Ihrer Dringlichkeit in Anlehnung an die „National Confidential Enquiry into Patient Outcome and Death“ - kurz NCEPOD-Klassifikation [10] eingestuft: Als Notfälle wurden jegliche Eingriffe mit vitaler Indikation (z. B. Stillung akuter, lebensbedrohlicher Blutungen; Debridement bei nekrotisierenden Weichteilinfekten) oder extremitätenerhaltende Eingriffe sowie verbrennungschirurgische Erstversorgungen klassifiziert. Operationen, die idealerweise innerhalb von maximal 12 Stunden durchgeführt werden sollten, z. B. Protheseninfekte, Handphlegmonen oder die Versorgung von Weichteilverletzungen ohne Notwendigkeit zur Revaskularisierung, wurden als akut gewertet. Als dringlich wurden Operationen zusammengefasst, die entweder aufgrund der jeweiligen Diagnose (z. B. onkologisch, chronische Osteomyelitis, Weichteildefekte, die einer plastisch-chirurgischen Deckung bedürfen), oder wegen der vorliegenden Beschwerden (z. B. Nervenkompressionssyndrome mit ausgeprägter Schmerzsymptomatik, funktionelle Einschränkungen mit signifikant schlechterem Outcome ohne zeitgerechte Therapie wie z. B. der 2. Akt einer Beugesehnenrekonstruktion) innerhalb von maximal 4 Wochen versorgt werden mussten. Alle übrigen Operationen (körperformende Eingriffe, funktionell-ästhetische Operationen, Narbenkorrekturen, aufwändige Rekonstruktionen bei lang vorbestehenden Deformitäten) wurden als elektiv eingestuft.

Um die Versorgungsqualität während des Covid-Lockdowns zu erheben, wurden zudem die Komplikationen erfasst und nach ihrer Schwere gemäß der Klassifikation nach Clavien-Dindo eingeteilt [11].

Die stationären Pflegetage und Intensivpflegetage (Anm.: Anzahl der Tage, die ein Patient stationär aufgenommen ist) wurden kumulativ für die jeweiligen Zeiträume erfasst, um die Auswirkung des Lockdowns auf die Bettenbelegung zu erheben.

Auf eine COVID-19-Infektion getestet wurden während des Untersuchungszeitraumes Patienten mit begründetem Verdacht, bei entweder entsprechender Symptomatik oder Exposition in der Anamnese.

Ebenso wurden ärztliche Mitarbeiter sowie das übrige Personal auf den chirurgischen Normalstationen nur im begründeten Verdachtsfall bzw. bei entsprechender Exposition getestet. Zusätzlich wurden Pflegekräfte, welche auf den COVID-Verdachtsstationen sowie auf den COVID-Isolierstationen eingesetzt wurden und permanent einem Infektionsrisiko ausgesetzt waren wöchentlich getestet.

\section{Statistische Methoden}

Die erhobenen Daten aus beiden Zeiträumen wurden miteinander verglichen. Die statistische Analyse wurde mit SPSS 24.0 für Windows (IBM Inc., Armonk, NY, USA) durchgeführt. Zur Testung auf Normalverteilung wurde der Levene-Test für Varianzgleichheit angewandt. Zur induktiven Analyse wurde bei dichotomen Para- 
Operationen je Woche, KW 10-17, 2019 vs. 2020

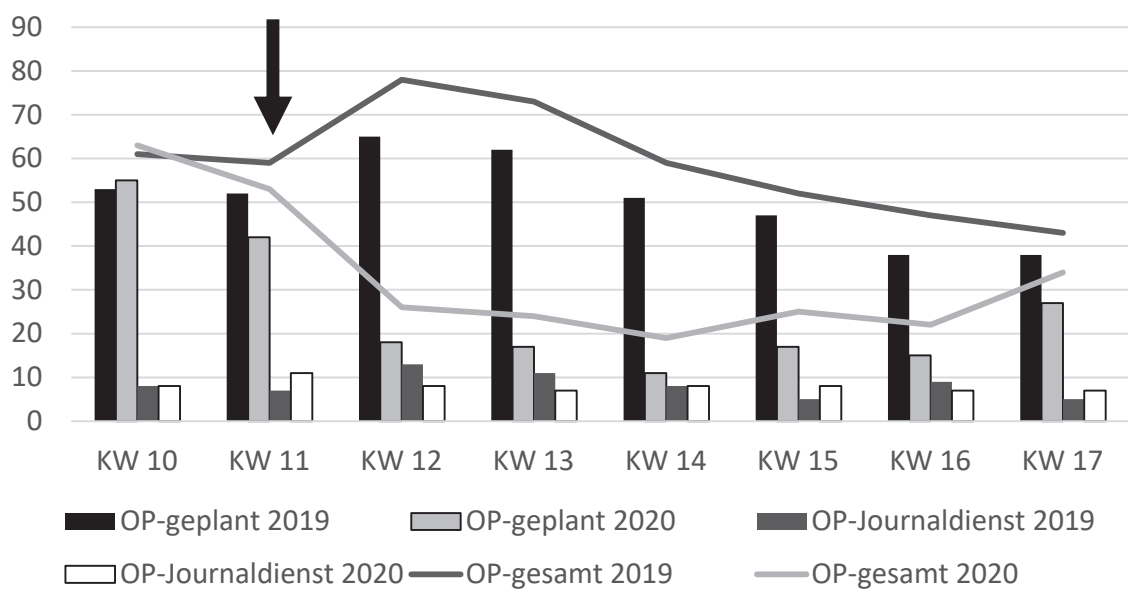

- Abb. 1 Summe der wöchentlichen Operationen 2019 und 2020 in Kalenderwoche (KW) 10 bis 17, aufgeteilt in Gesamtanzahl, geplante Operationen und Operationen im Bereitschaftsdienst. Der Pfeil markiert den Zeitpunkt des Lockdowns.

metern der Chi²-Test, bei ordinal skalierten Parametern wurde die Korrelation nach Spearman angewandt. Unterschiede bei kontinuierlichen, normalverteilten Parametern wurden mit dem T-Test ermittelt. In allen Fällen wurde ein p-Wert <0,05 als signifikant definiert. Die Ergebnisse sind in Mittelwert und Standardabweichung angegeben, außer anders angegeben.

\section{Genehmigung der Ethikkommission}

Die Studie wurde von der Ethikkommission der Medizinischen Universität Graz entsprechend der Deklaration von Helsinki genehmigt (EK-Nr.: 32-480 ex 19/20).

\section{Ergebnisse}

\section{Effekt der COVID-19-Pandemie auf die Behandlungszahlen}

Während des sechswöchigen Lockdowns (KW 12 bis 17) wurden 150 (2020) Eingriffe durchgeführt, was verglichen mit demselben Zeitraum von 2019 (353 Eingriffe) einer Reduktion um 57,5\% entspricht. 2020 wurden in KW 10 und 11 (=Zeit vor dem Lockdown) 116 Eingriffe, verglichen mit 119 Eingriffen im Jahr 2019 durchgeführt. Im Durchschnitt wurden in den KW 12 bis 17 im Jahr 2020 signifikant weniger Patienten pro Tag operiert (2019: 8,4 \$ 5,3, 2020: 3,6 $\pm 2,3$, p<0,001; s. - Abb. 1). Die durchschnittliche Anzahl an Operationen pro Tag der KW 10 und 11 in 2019 und 2020 ergab keinen signifikanten Unterschied (2019: 8,6 $\pm 5,3,2020: 8,3 \pm 5,5, p=0,890)$.

\section{Die Auswirkungen der COVID-19-Pandemie auf das Operationsspektrum}

Im Zuge des COVID-bedingten Lockdowns und dem Wegfall der elektiven Eingriffe kam es zu einer Umverteilung innerhalb des üblichen Behandlungsspektrums. Dadurch kam es zu einer Reduktion um 59,1 \% der handchirurgischen (2019: 87, 2020: 47 Fälle), 49,2\% der plastisch- rekonstruktiven Eingriffe (2019: 177, 2020: 90 Fälle) sowie 100 \% der köperformenden Eingriffe (2019: 76,
2020: 0 Fälle, $p=0,001)$. Die Anzahl der Verbrennungseingriffe veränderte sich nicht (s. > Tab. 1). Dies ergab anteilsweise signifikant mehr verbrennungschirurgische (2019: 3,7\%, 2020: 8,7\% $\mathrm{p}=0,021$ ) und mehr plastisch-rekonstruktive Eingriffe (60\% vs. 2019: 50,1\%, $202060 \%, p=0$,043). In Relation wurden auch mehr handchirurgische Eingriffe (2019: 24.6\%, 2020: $31.3 \%, p=0,121$ ) durchgeführt, allerdings war hier der Unterschied nicht signifikant (s. - Tab. 1). In den KW 10 und 11 wurden dahingegen im Jahresvergleich annähernd gleich viele verbrennungschirurgische, handchirurgische, körperformende und plastisch-rekonstruktive Eingriffe durchgeführt.

\section{Operationen nach Dringlichkeit}

In den KW 10 und 11 fanden sich im Jahresvergleich keine signifikanten Unterschiede hinsichtlich der Dringlichkeiten der durchgeführten Eingriffe. Signifikante Unterschiede zeigten sich in den KW 12 bis 17. 52,7\% der Eingriffe im Jahr 2019 waren elektiver Natur (196 Fälle). Im Jahr 2020 wurden signifikant mehr Notfall- und Akuteingriffe durchgeführt, sowohl absolut als auch in Relation zur Gesamtanzahl (2019: 41, 2020: 58 Fälle; 2019: 11,6\%, 2020: 38,7\%, $\mathrm{p}<0,001$ ); vor Inkrafttreten der Maßnahmen (KW 10 und 11) gab es keine signifikanten Unterschiede ( $p=0,087$; siehe $>$ Tab. 2$)$.

\section{Auswirkung der COVID-19-Pandemie auf Verletzungen und Verletzungsursachen}

Während in den KW 10 und 11 im Jahresvergleich nahezu gleich viele Verletzungsfälle einer plastisch-chirurgischen Versorgung bedurften (2019: 7 [5,6\%], 2020: 10 [8,6\%], p = 0,418), gab es während des Lockdowns im Vergleich zum Vorjahr sowohl relativ zur Gesamtzahl der Operationen als auch absolut mehr Verletzungsfälle. In den betreffenden 6Wochen wurden im Jahr 2019 44 (12,5\% der Gesamtoperationszahl) Verletzungen operativ versorgt, im Vergleich zu 65 (43,3\% der Gesamtoperationszahl) im Jahr $2020(p<0,001)$. Auffallend war außerdem die Verteilung der Verletzungsursachen während dieser Zeit: 67,7\% $(n=44)$ geschahen im privaten Umfeld, 7,7\% ( $n=5)$ waren Arbeitsunfälle und 24,6\% 


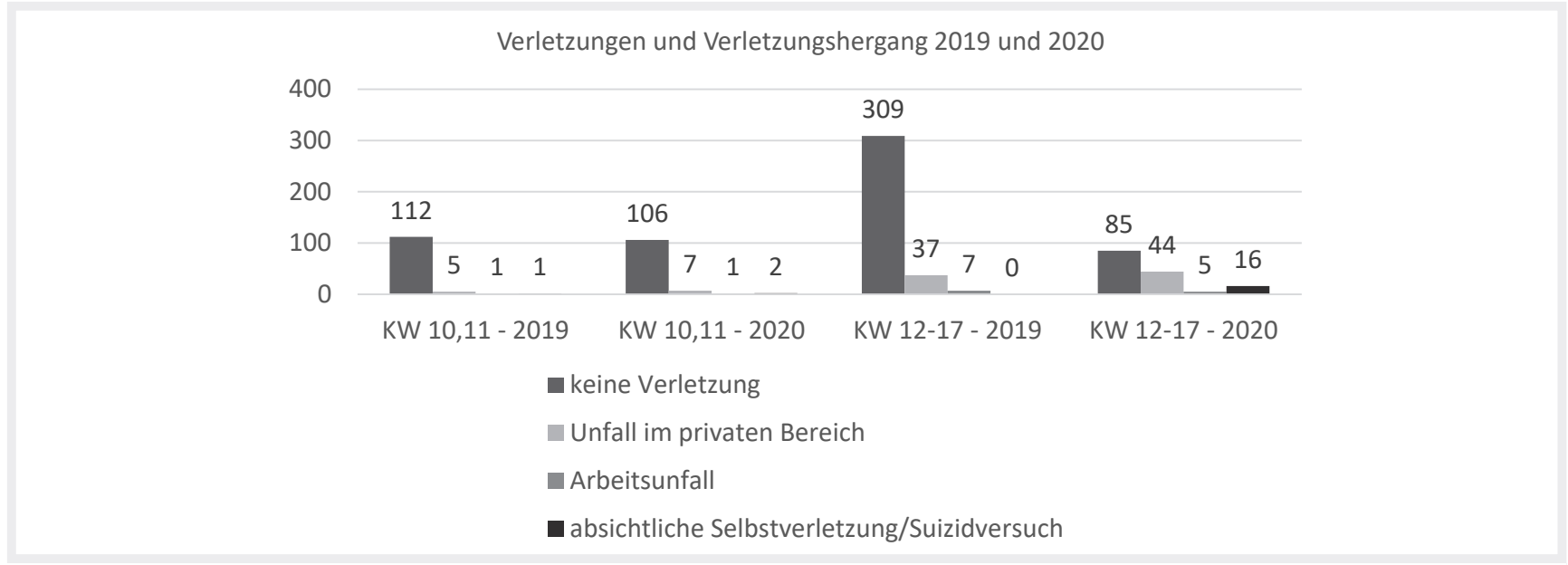

- Abb. 2 Verletzungen und deren Ursachen im Verhältnis zu den Gesamtbehandlungszahlen im Jahresvergleich, aufgeteilt nach Kalenderwoche (KW) 10 und 11 bzW. $12-17$.

> Tab. 1 Operationsspektrum vor (KW 10,1 11) und während (KW 12-17) des COVID-Lockdowns, im Jahresvergleich. Absolute und Prozente; Chi²-Test; signifikante p-Werte fett gedruckt; COVID-Zeitraum KW 12-17, KW = Kalenderwoche, J. = Jahr.

\begin{tabular}{|c|c|c|c|c|c|c|c|c|c|c|c|}
\hline \multirow[t]{2}{*}{ KW } & \multirow{2}{*}{ J. } & \multicolumn{2}{|c|}{ Verbrennungs-chirurgisch } & \multicolumn{2}{|c|}{ Handchirurgisch } & \multicolumn{2}{|c|}{ Körperformende Eingriffe } & \multicolumn{2}{|c|}{ Plastisch-Rekonstruktiv } & \multicolumn{2}{|c|}{ gesamt } \\
\hline & & 2019 & 2020 & 2019 & 2020 & 2019 & 2020 & 2019 & 2020 & 2019 & 2020 \\
\hline \multirow[t]{3}{*}{10,11} & $\mathrm{n}$ & 4 & 3 & 27 & 26 & 25 & 31 & 63 & 56 & 119 & 116 \\
\hline & $\%$ & $(3,4 \%)$ & $(2,6 \%)$ & $(22,7 \%)$ & $(22,4 \%)$ & $(21,0 \%)$ & $(26,7 \%)$ & $(52,9 \%)$ & $(48,3 \%)$ & $100 \%$ & $100 \%$ \\
\hline & $\mathrm{p}$ & \multicolumn{2}{|l|}{0,727} & \multicolumn{2}{|l|}{0,960} & \multicolumn{2}{|l|}{0,304} & \multicolumn{2}{|l|}{0,474} & & \\
\hline \multirow[t]{3}{*}{$12-17$} & $\mathrm{n}$ & 13 & 13 & 87 & 47 & 76 & 0 & 177 & 90 & 353 & 150 \\
\hline & $\%$ & $(3,7 \%)$ & $(8,7 \%)$ & $(24,6 \%)$ & $(31,3 \%)$ & $(21,5 \%)$ & $(0 \%)$ & $(50,1 \%)$ & $(60,0 \%)$ & $100 \%$ & $100 \%$ \\
\hline & $\mathrm{p}$ & \multicolumn{2}{|l|}{0,021} & \multicolumn{2}{|l|}{0,121} & \multicolumn{2}{|l|}{$<0,001$} & \multicolumn{2}{|l|}{0,043} & & \\
\hline
\end{tabular}

- Tab. 2 Dringlichkeit der Operationen vor (KW 10,11) und während (KW 12-17) des Lockdowns im Jahresvergleich, Absolute und Prozente; Korrelation nach Spearman, signifikante p-Werte fett gedruckt; COVID-Zeitraum KW 12-17, KW= Kalenderwoche, J. = Jahr.

\begin{tabular}{|c|c|c|c|c|c|c|c|c|c|c|}
\hline \multirow{2}{*}{ KW } & \multirow[b]{2}{*}{ J. } & \multicolumn{2}{|l|}{ Notfall } & \multicolumn{2}{|l|}{ Akut } & \multicolumn{2}{|l|}{ Dringlich } & \multicolumn{2}{|l|}{ Elektiv } & \multirow[t]{2}{*}{ p-Wert } \\
\hline & & 2019 & 2020 & 2019 & 2020 & 2019 & 2020 & 2019 & 2020 & \\
\hline \multirow[t]{2}{*}{10,11} & $\mathrm{n}$ & 1 & 4 & 13 & 10 & 34 & 18 & 71 & 84 & \multirow[t]{2}{*}{0,087} \\
\hline & $\%$ & $(0,9 \%)$ & $(3,4 \%)$ & $(10,9 \%)$ & $(8,6 \%)$ & $(28,6 \%)$ & $(15,5 \%)$ & $(59,7 \%)$ & $(72,4 \%)$ & \\
\hline \multirow[t]{2}{*}{$12-17$} & $\mathrm{n}$ & 6 & 13 & 35 & 45 & 116 & 79 & 196 & 13 & \multirow[t]{2}{*}{$<0,001$} \\
\hline & $\%$ & $(1,7 \%)$ & $(8,7 \%)$ & $(9,9 \%)$ & $(30,0 \%)$ & (32,9\%) & (54,3\%) & (52,7\%) & $(8,7 \%)$ & \\
\hline
\end{tabular}

( $n=16)$ geschahen in Zusammenhang mit absichtlicher Selbstverletzung oder Suizidversuchen. Demgegenüber geschahen im selben Zeitraum im Jahr 2019 84,1 \% ( $n=37)$ aller Verletzungen im privaten Umfeld ( $p=0,055), 15,9 \%(n=7)$ waren Arbeitsunfälle $(p=0,179)$, und es gab keine absichtlichen Selbstverletzungen oder Suizidversuche $(p<0,001)$. In den KW 10 und 11 gab es im Jahresvergleich bezüglich der Ursachenverteilung keine signifikanten Unterschiede (Unfälle im privaten Bereich: $p=0,949$, Arbeitsunfälle: $p=0,787$, absichtliche Selbstverletzung/Suizidversuch: $\mathrm{p}=0,761$; siehe $>$ Abb. 2 ).

\section{Komplikationsraten}

Im Vergleich zu 2019 bestand 2020 kein Unterschied hinsichtlich der operativen Komplikationsrate sowohl in der Zeit vor (KW 10 und $11 ; 2019: 10,9 \%, 2020: 6,0 \%, p=0,179)$ als auch während des COVID-Lockdowns (KW 12-17; 2019: 6,8\%, 2020: 10\%, p=0,219). Auch hinsichtlich der Komplikationsschwere gemäß der ClavienDindo-Klassifikation ergaben sich keine signifikanten Unterschiede (siehe $>$ Tab. 3 ). 
- Tab. 3 Demographische Parameter, Anzahl der Eingriffe und Komplikationsraten vor (KW 10,11) und während (KW 12-17) des Lockdowns im Jahresvergleich. Unterschiede im ASA Status und bei Komplikationen klassifiziert nach Clavien-Dindo wurden mittels Spearman-Korrelation verglichen.KW = Kalenderwoche, SD = Standardabweichung; Der ASA-Status ist nur zu Patienten vorhanden, die in Plexus- oder Allgemeinanästhesie operiert wurden - bei Patienten, die in Lokalanästhesie operiert wurden oder bei Notfällen wurde der ASA-Status nicht routinemäßig erhoben; signifikante p-Werte fett gedruckt; COVID-Zeitraum KW 12-17.

\begin{tabular}{|c|c|c|c|c|}
\hline KW & & 2019 & 2020 & p-Wert \\
\hline \multirow[t]{14}{*}{10,11} & Männer, n (\%) & $45(37,8 \%)$ & $50(43,1 \%)$ & 0,409 \\
\hline & Alter, Durchschnitt $( \pm S D)$ & $46,8( \pm 20,7)$ & $46,5( \pm 18,5)$ & 0,892 \\
\hline & Operationen gesamt & 119 & 116 & \\
\hline & ASA-Status vorhanden ${ }^{\mathrm{a}}$ & $81(68,1 \%)$ & $77(66,4 \%)$ & \\
\hline & ASA 1 & $26(32,1 \%)$ & $19(24,7 \%)$ & \multirow[t]{4}{*}{0,077} \\
\hline & ASA 2 & $42(51,9 \%)$ & $36(46,8 \%)$ & \\
\hline & ASA 3 & $12(14,8 \%)$ & $19(24,7 \%)$ & \\
\hline & ASA 4 & $1(1,2 \%)$ & $3(3,9 \%)$ & \\
\hline & Komplikationen & $13(10,9 \%)$ & $7(6,0 \%)$ & 0,179 \\
\hline & Clavien-Dindo I & $1(7,7 \%)$ & $1(14,3 \%)$ & \multirow[t]{5}{*}{0,177} \\
\hline & Clavien-Dindo II & $0(0 \%)$ & $0(0 \%)$ & \\
\hline & Clavien-Dindo IIla & $1(7,7 \%)$ & $2(28,6 \%)$ & \\
\hline & Clavien-Dindo IIIb & $10(76,9 \%)$ & $4(57,1 \%)$ & \\
\hline & Clavien-Dindo IV & $1(7,7 \%)$ & $0(0 \%)$ & \\
\hline \multirow[t]{14}{*}{$12-17$} & Männer, n (\%) & $176(49,9 \%)$ & $94(62,7 \%)$ & 0,008 \\
\hline & Alter, Durchschnitt ( \pm SD) & $50,0( \pm 20,4)$ & $50,7( \pm 18,5)$ & 0,687 \\
\hline & Operationen gesamt & 353 & 150 & \\
\hline & ASA-Status vorhandena & $178 / 353(50,4 \%)$ & $120 / 150(80,0 \%)$ & \\
\hline & ASA 1 & $61(34,3 \%)$ & $22(18,3 \%)$ & \multirow[t]{4}{*}{0,001} \\
\hline & ASA 2 & $71(39,9 \%)$ & $54(45,0 \%)$ & \\
\hline & ASA 3 & $40(22,5 \%)$ & $31(25,8 \%)$ & \\
\hline & ASA 4 & $6(3,4 \%)$ & $13(10,8 \%)$ & \\
\hline & Komplikationen & $24(6,8 \%)$ & $15(10,0 \%)$ & 0,219 \\
\hline & Clavien-Dindo I & $10(41,7 \%)$ & $2(13,3 \%)$ & \multirow[t]{5}{*}{0,842} \\
\hline & Clavien-Dindo II & $0(0 \%)$ & $4(26,7 \%)$ & \\
\hline & Clavien-Dindo IIla & $1(4,2 \%)$ & $3(20 \%)$ & \\
\hline & Clavien-Dindo IIIb & $13(54,2 \%)$ & $6(40 \%)$ & \\
\hline & Clavien-Dindo IV & $0(0 \%)$ & $0(0 \%)$ & \\
\hline
\end{tabular}

\section{Demographische Unterschiede vor und während des COVID-Lockdowns}

Vor dem Lockdown bestanden im Jahresvergleich keine signifikanten Unterschiede hinsichtlich Alters-, Geschlechtsverteilung oder ASA-Status der Patienten. Während des Lockdowns wurden im Vergleich zu 2019 im Verhältnis signifikant mehr Männer operiert (2019: 176 [49,9\%], 2020: 94 [62,7\%], p = 0,008); die Altersverteilung blieb hingegen gleich (2019: 50,0 $\pm 20,4,2020$ : 50,7 $\pm 18,5$, $p=0,687)$. Außerdem waren ASA-Grade $>1$ während des Lockdowns signifikant häufiger ( $p=0,077$; siehe $>$ Tab. 3 ).

\section{Auswirkung der COVID-19-Pandemie auf die Bettenbelegung}

In den Kalenderwochen 10 und 11 im Jahr 2019 beliefen sich die stationären Pflegetage aller Patienten auf insgesamt 396, während im Jahr 2020395 Pflegetage verzeichnet wurden. Im Jahr 2020 war dabei der Anteil der Intensivpflegetage bereits vor dem Lockdown signifikant höher als im Jahr 2019 (34 [8,6\%], 3 [0,8\%], p<0,001). In den Kalenderwochen 12 bis 17 wurden im Jahr 2020 mit 715 Pflegetagen deutlich weniger Pflegetage als im Jahr 2019 (2935 Tage) verzeichnet. Der Anteil der Intensivpflegetage war im Jahr 2020 signifikant höher als im Jahr 2019 (61 [8,5\%], 5 [0,2\%], p<0,001, siehe $>$ Tab. 4).

\section{COVID-19-Verdachtsfälle und tatsächlich positiv getestete Patienten}

Bei den insgesamt 150 während des Lockdowns durchgeführten Operationen bestand in 19 Fällen (12,7\%) der Verdacht auf eine COVID-19-Infektion, welcher sich bei lediglich 2 Patienten (mit insgesamt 4 Operationen) bestätigte. Diese Operationen wurden unter Einhaltung sämtlicher empfohlenen COVID-Schutzmaßnahmen durchgeführt [12]. 
- Tab. 4 Verteilung der Pflege- und Intensivpflegtage vor (KW 10, 11) und während (KW 12-17) des Lockdowns bzw. im Vergleich zwischen 2019 und 2020. Normal = Pflegetage auf Normalstation, Intensiv = Pflegetage auf Intensivstation.

\begin{tabular}{|l|l|l|l|l|}
\hline & Pflegetage & $\mathbf{2 0 1 9}$ & $\mathbf{2 0 2 0}$ & p-Wert \\
\hline \multirow{2}{*}{ KW 10, 11 } & Normal & $393(99,2 \%)$ & $361(91,4 \%)$ & $<0,001$ \\
\cline { 2 - 4 } & Intensiv & $3(0,8 \%)$ & $34(8,6 \%)$ & \\
\cline { 2 - 4 } & gesamt & $396(100 \%)$ & $395(100 \%)$ & - \\
\hline \multirow{2}{*}{ KW 12-17 } & Normal & $2930(99,8 \%)$ & $654(91,5 \%)$ & $<0,001$ \\
\cline { 2 - 4 } & Intensiv & $5(0,2 \%)$ & $61(8,5 \%)$ & \\
\cline { 2 - 4 } & gesamt & $2935(100 \%)$ & $715(100 \%)$ & - \\
\hline
\end{tabular}

\section{Diskussion}

In der vorliegenden Studie wurden die Auswirkungen der COVID19-Pandemie auf das Operationsspektrum der klinischen Abteilung für Plastische, Ästhetische und Rekonstruktive Chirurgie der Universitätsklinik für Chirurgie an der medizinischen Universität Graz untersucht. Insgesamt war die Gesamtzahl der Operationen durch den Lockdown signifikant reduziert (minus 57,5\%). Die wochenweise Analyse zeigte, dass der Rückgang der OP-Zahlen in erster Linie durch die Reduktion des geplanten Regelprogramms zustande kam, während die Anzahl der Operationen zur Bereitschaftsdienstzeit im Vergleich der beiden Jahre konstant blieb. Dies betont die Wichtigkeit der plastisch-chirurgischen Dienstärzte vor Ort außerhalb der Regeldienstzeit. Zu einem ähnlichen Ergebnis kam eine Arbeit einer deutschen Universitätsklinik. Bernuth et al. konnten eine Auslastung der OP-Kapazität von 57,3\% im Vergleich zum Regelbetrieb während des Lockdowns zeigen [13]. Dies spiegelt einen noch geringeren Anteil an Elektivoperationen an dieser Klinik wider. Bei Notfalleingriffen und dringlichen Fällen, was unserer Einteilung nach auch Akuteingriffe beinhaltet, zeigte sich in dieser Arbeit kein signifikanter Unterschied im Jahresvergleich, Berücksichtigt man diese abweichende Einteilung, sind diese Ergebnisse mit denen unserer Studie vergleichbar.

\section{Die plastische Chirurgie als Domäne in der Versorgung akuter und dringlicher Fälle}

Typischerweise ist ein signifikanter Anteil der Eingriffe im Fach der Plastischen, Ästhetischen und Rekonstruktiven Chirurgie elektiver Natur. Dazu zählen einerseits alle ästhetischen bzw. körperformenden Eingriffe, jedoch auch die meisten komplexen rekonstruktiven Eingriffe [14]. Dass in unserer Untersuchung rund die Hälfte aller handchirurgischen- und plastisch-rekonstruktiven und alle körperformenden Eingriffe wegfielen, bestätigt dies. Das Aussetzten elektiver Operationen zur Schaffung allfällig benötigter Kapazitäten im Rahmen der COVID-19 Pandemie ermöglichte es, unser Team im Sinne des Infektionsschutzes für diesen Zeitraum auf rund die Hälfte zu reduzieren und für die nicht anwesenden Kollegen eine prophylaktische Schutzisolation anzuordnen; dadurch hätte im Falle einer COVID-19 Erkrankung eines Teammitgliedes das zweite Team einberufen werden können [12]. Die Effektivität dieser und anderer Maßnahmen wurde dadurch bestätigt, dass das gesamte Department für Chirurgie an unserer Klinik, keinen einzigen COVID-
19 positiven Mitarbeiter, weder seitens der Pflege, noch ärztlicherseits zu verzeichnen hatte.

Die absolute Anzahl der Notfalleingriffe und der Akuteingriffe stieg während des Lockdowns signifikant an. Unter strenger Abwägung von Nutzen- und Risiko wurden auch dringliche Operationen wie Tumorresektionen und konsekutive Rekonstruktionen durchgeführt. Auch wurden einige prinzipiell als elektiv einzustufende Operationen durchgeführt. Der Grund dafür ist, dass Operationen wie z. B. eine primäre Nervenrekonstruktion nach traumatischer Plexusverletzung zeitgerecht durchzuführen sind, weswegen in Einzelfällen trotz elektiver Indikation die Operationsindikation gestellt wurde, um eine Verschlechterung des Ergebnisses bei diesen Patienten zu verhindern [15]. Auch Operationen wie die Durchtrennung von Lappenstielen (Stirnlappen, Cross-Finger-Lappen), welche prinzipiell als elektiv einzustufen sind, wurden als dringlich gewertet und zeitnah durchgeführt, um einer Verschlechterung des Ergebnisses (z. B. durch eine Wundinfektion) oder Sekundärschäden (z. B. durch prolongierte Immobilisation) vorzubeugen.

\section{Verletzungen und Verletzungsursachen während des Lockdowns}

Es kam zu einer signifikanten Zunahme von chirurgisch zu versorgenden Verletzungen. Im privaten Bereich sahen wir vermehrt Unfälle durch handwerkliche Tätigkeiten, wenn auch nicht statistisch signifikant. Des Weiteren kam es zu einem signifikanten Anstieg von Selbstverletzungen bzw. Suizidversuchen. Ersteres kann auf die reduzierte Arbeitszeit inklusive Kurzarbeit während des Lockdowns in Verbindung mit der frühen Wiedereröffnung von Baumärkten [16], letzteres auf die massive psychische Belastung und subjektiv als bedrohlich empfundene Pandemie zurückgeführt werden [17]. Interessanterweise gab es keinen signifikanten Rückgang im Bereich der Arbeitsunfälle trotzt vorübergehender starker Einschränkung diverser Wirtschaftszweige inklusive Produktion und Fertigung und einer vorübergehenden Einstellung von Baustellen [18]. Das kann dadurch erklärt werden, dass an untersuchter Abteilung generell wenig Arbeitsunfälle versorgt werden, da diese Patienten in Österreich überwiegend an den Unfallkrankenhäusern (ähnlich den deutschen BG Kliniken) versorgt werden.

\section{Behandlungsqualität während des Lockdowns}

Die Komplikationsrate änderte sich während des Lockdowns nicht signifikant. Dies bedeutet zum einen, dass der Anteil elektiver Eingriffe keinen Einfluss auf die Komplikationsrate zu haben scheint, und andererseits, dass Notfall-, Akut- und dringliche Operationen während des Lockdowns ohne signifikanten Anstieg der Komplikationsrate durchgeführt werden konnten. Damit konnte gezeigt werden, dass trotz sämtlicher Beschränkungen während der Pandemie eine qualitativ konstante Patientenversorgung auf höchstem Niveau gewährleistet war. Es gilt zu evaluieren, ob im Falle einer neuerlichen Zunahme der Infektionszahlen sämtliche elektiven Operationen wieder ausgesetzt werden müssen, oder unter Einhaltung entsprechender Schutzvorkehrungen durchgeführt werden können.

Dies muss auch bei ästhetischen Eingriffen diskutiert werden. Doch es erscheint in jedem Fall sinnvoll, dass elektive Fälle, welche die Lebensqualität der Patienten stark einschränken (z. B. Ringbandstenosen oder rekonstruktive Handeingriffe) oder bei denen 
es möglicherweise sogar zu einem schlechteren Langzeitergebnis kommen kann (z. B. progrediente Nervenkompressionssyndrome) oder in besonderen Fällen auch körperformende Eingriffe mit kurzer Liegedauer, unter Einhaltung entsprechenden Schutzmaßnahmen für Patienten und Personal dennoch durchgeführt werden sollten. Bei Wiederanstieg der Infektionsrate könnte man rasch handeln und innerhalb kürzester Zeit wieder auf den „Pandemiebetrieb“ umstellen [12].

\section{COVID-19 positive Patienten}

Zum Zeitpunkt der Einreichung des Manuskripts war lediglich bei 2 Patienten (Versorgung einer Selbstverletzung durch eine Schrotflinte, Wundheilungsstörung nach offener Unterschenkelfraktur) eine COVID-19 Infektion nachgewiesen worden. Die insgesamt 4 Operationen wurden unter Einhaltung sämtlicher empfohlener Sicherheitsmaßnahmen durchgeführt [12]. Es gab an unserer Abteilung keine Komplikation, die auf eine COVID-19-Infektion zurückzuführen war. Die Kapazitäten des Zentrums und seiner Spezialdisziplinen wurde weitgehend für Patienten freigehalten, für welche eine Behandlung in anderen Krankenhäusern aufgrund der Komplexität nicht in Frage kam. COVID-19-Verdachtsfälle und -positive Patienten ohne Zentrumsindikation wurden nach Möglichkeiten in einem peripheren Krankenhaus behandelt, Nachkontrollen weitestgehend in den niedergelassenen Bereich oder in periphere Spitalsambulanzen ausgelagert. Dadurch wurde das Risiko einer COVID-19-Infektion innerhalb des Klinikums reduziert [12].

Der ASA Status der während des Lockdowns operierten Patienten war im Jahresvergleich höher. Das weist darauf hin, dass die durchschnittliche Morbidität der während dieser Zeit operierten Patienten höher war bzw. diese häufiger relevante Nebendiagnosen aufwiesen, somit insgesamt kranker waren (siehe - Tab. 3). Nachdem bei multimorbiden Patienten nach Möglichkeit keine elektiven Eingriffe durchgeführt werden, ist diese Beobachtung zumindest teilweise auf den Wegfall der Elektiveingriffe zurückzuführen. Umso bemerkenswerter ist, dass es trotz häufigerer Komorbidität zu keinem signifikanten Anstieg der Komplikationsrate kam.

\section{Zeit nach dem Lockdown - Aufarbeiten des Operationsrückstaus}

Während Tumoreingriffe, die streng genommen gut planbar sind, aber unter gewissem Zeitdruck durchgeführt werden müssen durch den Lockdown nicht betroffen waren, musste eine Vielzahl plastischer Eingriffe, wie z. B. komplexe Brustrekonstruktionen, körperformende Eingriffe oder geschlechtsangleichende Operationen aufgrund des operativen Aufwands verschoben werden. Darüber hinaus wurden auch nicht-dringliche Eingriffe bei multimorbiden Patienten unter Berücksichtigung des Infektionsrisikos eines Krankenhausaufenthalts während der Pandemie vorläufig verschoben, so z. B. Dekubitalulzera oder chronische Schmerzsyndrome. Zum gegenwärtigen Zeitpunkt kann über die Auswirkungen dieser Maßnahmen auf die physische und psychische Gesundheit der betroffenen Patienten keine Aussage getroffen werden.

Umso mehr gilt es die verschobenen Operationen zeitgerecht nachzuholen, um sogenannte medizinische „Kollateralschäden“ zu vermeiden und langfristige negative Auswirkungen auf die Morbidität und Mortalität dieser Patienten zu minimieren [19]. Hierfür wird nicht nur eine - zumindest vorübergehende - Erweiterung der
Operationskapazitäten nötig sein; es wird auch einer Steigerung der Bettenanzahl und der Ambulanzkapazität zur perioperativen Planung und postoperativen Nachbehandlung bedürfen. Dies könnte beispielsweise durch Verlängerung des Routinebetriebs eines OPTisches bis zum Abend und entsprechender Schichtbesetzung ermöglicht werden. Allerdings ist eine grundsätzliche Erhöhung von Versorgungskapazitäten bei grundsätzlich eher hoher Auslastung der Abteilung nicht einfach umzusetzen, sodass davon auszugehen ist, dass entsprechend ausgefallene Eingriffe als zusätzlicher Faktor im Regelprogramm über längere Zeit berücksichtigt werden müssen. In einem Konsensbeschluss der European Association of Societies of Aesthetic Surgery (EASAPS) und der European Society of Plastic, Reconstructive and Aesthetic Surgery (ESPRAS) wurden Empfehlungen hinsichtlich einer sicheren Wiederaufnahme des Regelbetriebs als auch bezüglich des Vorgehens im Falle einer erneuten Infektionswelle definiert [20]. Diese Empfehlungen wurden selbstverständlich bei der Planung der Wiederaufnahme des Regelbetriebs an unserer Abteilung berücksichtigt. Einen Überblick über Möglichkeiten, den Regelbetrieb in einem Krankenhaus der Maximalversorgung für Personal und Patienten sicher und ressourcenschonend wieder aufzunehmen, haben Kricheldorff et al. in einer fundierten Übersichtsarbeit dargestellt [21]. Die darin angewandte Behandlungstriage unterscheidet sich etwas von der Einteilung im Rahmen der Aufarbeitung unserer Daten, erscheint jedoch im Hinblick auf Praktikabilität im Falle eines erneuten Aussetzens des Elektivprogramms als auch bei der Abarbeitung der angehäuften Fälle sehr hilfreich. Auch die vermehrte Anwendung von Telemedizin, insbesondere in der Sprechstunde, scheint in der aktuellen Situation und in Zukunft von großem Vorteil. Hierfür ist es notwendig auch in Österreich entsprechende Rahmenbedingungen zu schaffen.

\section{Limitationen}

Unsere Daten sind auf den Zeitraum des Lockdowns, einschließlich eines kurzen Zeitfensters davor beschränkt, weswegen unsere Ergebnisse lediglich eine Momentaufnahme repräsentieren. Aussagen über Auswirkungen durch den Wegfall elektiver Operationen können zum Zeitpunkt der Fertigstellung der Arbeit noch nicht getroffen werden.

\section{Schlussfolgerung}

Die Ergebnisse haben die Relevanz in der Krankenversorgung und den Beitrag, den die Plastische Chirurgie dabei leistet, aufgezeigt. Insbesondere ist hervorzuheben, dass ein wesentlicher Anteil der plastisch-chirurgischen Operationen aus Notfalleingriffen und dringenden, medizinisch indizierten Operationen besteht. Der Wegfall der elektiven Operationen hat zu einer Reduktion des OP-Programms auf etwa $42 \%$ der Vollauslastung geführt. Im Rückschluss darauf sind über $40 \%$ der Operationen der medizinischen Akutversorgung zuzuordnen. Allerdings ist dies umgekehrt nicht gleichbedeutend damit, dass elektive Operationen aus medizinischer Sicht als nicht notwendig angesehen werden können. Vielmehr ist „elektiv“ in diesem Zusammenhang gleichbedeutend damit, dass der jeweilige Eingriff medizinisch indiziert ist, aber vorübergehend auf- 
geschoben werden kann, ohne dass dabei ein Nachteil für den Patienten zu erwarten ist.

Insbesondere in Zeiten einer Pandemie gilt es die dafür notwendigen personellen und materiellen Ressourcen zur Verfügung zu stellen, um die Versorgung der Patienten rund um die Uhr zu gewährleisten. Um weiterhin eine Versorgung plastisch-chirurgischer Patienten auf höchstem Niveau sicherzustellen zu können und negative Folgen für Patienten abzuwenden, ist eine vorübergehende Aufstockung der Operationskapazität inklusive Betten- und Ambulanzkapazität notwendig. Für mögliche zukünftige Pandemien ist die Bevölkerung ausreichend über Vermeidung von risikoreichen Verhalten aufzuklären, um Freizeitunfälle wie sie beim Heimwerken auftreten zu reduzieren. Sollte, wie aktuell möglicherweise in Teilen Chinas und Japans in Entstehung, eine zweite Welle auftreten, sollte diskutiert werden inwieweit ein erneuter absoluter Lockdown notwendig und sinnvoll ist, oder ob Risikogruppen anderweitig ausreichend geschützt werden können. Insbesondere sollten Kosten und Nutzen sorgfältig abgewogen werden - sowohl im Hinblick auf die medizinische Versorgung als auch aus gesamtökonomischer Sicht auf mögliche Folgen auf die physische- und psychische Gesundheit.

Interessenkonflikt

Die Autoren erklären, dass kein finanzieller Interessenskonflikt besteht. Der Inhalt des Artikels wurde ausschließlich von den gelisteten Autoren geschrieben. Bei keinem der Autoren besteht ein Interessenskonflikt, eine Bindung oder finanzielle Beteiligung an einer Organisation mit finanziellem Interesse oder Konflikt mit dem behandelten Thema oder hier diskutierten Materialien.

\section{Autorinnen/Autoren}

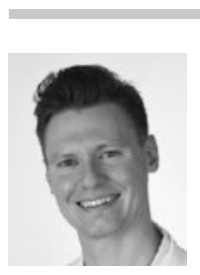

\section{Dr. med. univ. Daniel Popp}

Nach der Matura am Oeverseegymnasium Graz absolvierte Dr.med. univ. Popp das Medizinstudium an der Medizinischen Universität Graz mit Studienaufenthalten in Neuseeland, Mexiko und Barcelona. Teile des praktischen Jahres absolvierte er in München und in Luzern. Nach zweijähriger chirurgischer Basisausbildung in der Schweiz und weiteren 2 Jahren klinischer Tätigkeit an der Abteilung für Plastische, Rekonstruktive und Ästhetische Chirurgie am Ameos Klinikum Bremerhaven Mitte, absolvierte er einen zweijährigen Forschungsaufenthalt in den USA als Postdoctoral Research Fellow bei Dr. David N. Herndon bzw. Dr. Steven E. Wolf am Department of Surgery, University of Texas Medical Branch und am Shriners Hospitals for Children in Galveston, Texas. Seit Ende 2019 ist Dr. Popp an der Klinischen Abteilung für Plastische, Ästhetische und Rekonstruktive Chirurgie an der Universität Graz bei Professor Kamolz tätig. Sein besonderes Interesse gilt der Wiederherstellungschirurgie und der Verbrennungschirurgie.

\section{Referenzen}

[1] Bundesministerium für Digitalisierung und Wirtschaftsstandort. Allgemeine Maßnahmen (15.3.2020). Im Internet: https://www. oesterreich.gv.at/themen/coronavirus_in_oesterreich/weitere_ ma\%C3\%9Fnahmenb_bek\%C3\%A4mpfung_coronavirus.html; Stand 20.6.2020
[2] Giunta RE, Frank K, Costa H et al. The COVID-19 Pandemic and its Impact on Plastic Surgery in Europe - An ESPRAS Survey. Handchir Mikrochir Plast Chir 2020; 52: 221-232. doi:10.1055/a-1169-4443

[3] COVIDSurg Collaborative. Elective surgery cancellations due to the COVID-19 pandemic: global predictive modelling to inform surgical recovery plans. Br J Surg 2020. Anm.: Online ahead of print. doi:10.1002/bjs.11746. Online ahead of print.

[4] Stafford N. Covid-19: Why Germany's case fatality rate seems so low. BM] 2020; 369. doi:10.1136/bmj.m1395

[5] de Blacam C, Kilmartin D, Mc Dermott C et al. Public perception of Plastic Surgery. J Plast Reconstr Aesthet Surg 2015; 68: 197-204. doi:10.1016/j.bjps.2014.10.008

[6] Lifchez SD, Friedrich JB, Hultman CS. The scope of practice of hand surgery within plastic surgery: the ACAPS national survey to assess current practice and develop educational guidelines. Ann Plast Surg 2015; 74: 89-92. doi:10.1097/SAP.0000000000000365

[7] Peterson SL, Moore EE. The integral role of the plastic surgeon at a level I trauma center. Plast Reconstr Surg 2003; 112: 1371-1378. doi:10.1097/01.PRS.0000082815.79881.51

[8] Vrouwe SQ, Pham CH, Minasian RA et al. The State of Burn Care Training During Plastic Surgery Residency. Ann Plast Surg 2020; 85: 122-126. doi:10.1097/SAP.0000000000002267

[9] Giunta RE, Frank K, Moellhoff N et al. The COVID-19 Pandemia and its consequences for plastic surgery and hand surgery. Handchir Mikrochir Plast Chir 2020; 52: 233-241. doi:10.1055/a-1163-9009

[10] National Confidential Enquiry into Patient Outcome and Death. Classification of Intervention. Im Internet: https://www.ncepod.org.uk/ classification.html; Stand 24.6.2020

[11] Clavien PA, Barkun J, de Oliveira ML et al. The Clavien-Dindo classification of surgical complications: five-year experience. Ann Surg 2009; 250: 187-196. doi:10.1097/SLA.0b013e3181b13ca2

[12] Nischwitz SP, Popp D, Sawetz I et al. Burns in pandemic times - The Graz way towards COVID-19 and back (17.6.2020). Burns 2020. Online ahead of print. Im Internet: http://www.sciencedirect.com/science/ article/pii/S0305417920304277; Stand 17.6.2020

[13] Bernuth S, Horch RE, Vater A et al. [The impact of SARS-CoV-2 restrictions on medical care in Plastic Surgery]. Handchir Mikrochir Plast Chir 2020; 52: 272-279. doi:10.1055/a-1220-6920

[14] American Society of Plastic Surgeons. 2018 Plastic Surgery Statistics Report (20.6.2020). Plastic Surgery 2018. Im Internet: https://www. plasticsurgery.org/documents/News/Statistics/2018/plastic-surgerystatistics-full-report-2018.pdf; Stand 20.6.2020

[15] Rankine JJ. Adult traumatic brachial plexus injury. Clinical Radiology 2004; 59: 767-774. doi:10.1016/j.crad.2004.03.014

[16] ORF Österreichischer Rundfunk. Ansturm nur auf Baumärkte (14.4.2020); 2020. Im Internet: https://wien.orf.at/stories/3043870/; Stand 7.5.2020

[17] Gunnell D, Appleby L, Arensman E et al. Suicide risk and prevention during the COVID-19 pandemic. The Lancet Psychiatry 2020; 7 : 468-471. doi:10.1016/S2215-0366(20)30171-1

[18] Österreichischer Kommunalbau. Corona-Pandemie: Beton- und Fertigteilwerke fahren Produktion zurück (18.3.2020). Im Internet: https://a3bau.at/corona-pandemie-beton-und-fertigteilwerkefahren-produktion-zurueck; Stand 7.5.2020

[19] Kamolz L-P, Spendel S. The COVID-19 Pandemic and its Consequences for Plastic Surgery and Hand Surgery: a comment from the Graz University Hospital. Handchir Mikrochir Plast Chir 2020; 52: 242-243. doi:10.1055/a-1165-6799

[20] van Heijningen I, Frank K, Almeida F et al. EASAPS/ESPRAS Considerations in getting back to work in Plastic Surgery with the COVID-19 Pandemic - A European point of view. Handchir Mikrochir Plast Chir 2020; 52 (4): 257-264. doi:10.1055/a-1175-4169

[21] Kricheldorff], Strempel M, Hetmank C et al. [Plastic Surgery in times of COVID-19 in a maximum care hospital in Berlin - Description of the situation and approaches to action for resumption of regular operation]. Handchir Mikrochir Plast Chir 2020; 52: 265-271. doi:10.1055/a-1209-3122 